\title{
The Efficiency of the Biweight as a Robust Estimator of Location
}

\author{
Karen Kafadar* \\ National Bureau of Standards, Washington, DC 20234
}

November 9, 1982

\begin{abstract}
The biweight is one member of the family of M-estimators used to estimate location. The variance of this estimator is calculated via Monte Carlo simulation for samples of sizes 5, 10, and 20 . The scale factors and tuning constants used in the definition of the biweight are varied to determine their effects on the variance. A measure of efficiency for three distributional situations (Gaussian and two stretched-tailed distributions) is determined. Using a biweight scale and a tuning constant of $c=6$, the biweight attains an efficiency of $98.2 \%$ for samples of size 20 from the Gaussian distribution. The minimum efficiency at $n=$ 20 using the biweight scale and $c=4$ is $84.7 \%$, revealing that the biweight performs well even when the underlying distibution of the samples has abnormally stretched tails.
\end{abstract}

Key words: bisquare weight function; biweight scale estimate; median absolute deviation; M-estimator; tuning constant.

\section{Introduction}

Robust estimation of location has become an important tool of the data analyst, due to the recognition among statisticians that parametric models are rarely absolutely precise. Much discussion has taken place to determine the "best" estimators ("best" in a certain sense, such as low variance across several distributional situations). Estimators which were designed to be robust against departures from the Gaussian distribution in a symmetric, long-tailed fashion were investigated indepth by Andrews et al. in 1970-1971 [1]. ${ }^{1}$ Subsequent to this, Gross and Tukey compared several other estimators in the same fashion, one of which they called the biweight [2]. It was designed to be highly efficient in the Gaussian situation as well as in other symmetric, long-tailed situations. The first reference of its practical use appears two years later [3]. Gross showed that the biweight proves useful in the " $t$ "-like confidence interval for the one-sample problem [4] and for estimating regression coefficients [5]; Kafadar showed that it is efficient for the two-sample problem also [6].

\footnotetext{
*Center for Applied Mathernatics, National Engineering Laboratory.
}

'Figures in brackets indicate literature references at the end of this paper.
Many scientists collect data and perform elementary statistical analyses but seldom use summary statistics other than the sample mean and sample standard deviation. This paper is therefore addressed to two audiences. It provides a brief introduction to the field of robust estimation of location to explain the biweight in particular (section 2). Those who are familiar with the basic concepts may wish to proceed directly to section 3 which raises the specific questions about the biweight's computation and efficiency that are answered in this paper. Section 4 describes the results of a Monte Carlo evaluation of the biweight. An example to illustrate the biweight calculation is presented in section 5 , followed by a summary in section 6 .

\section{Robust Estimation of Location; M-Estimates.}

Given a random sample of $n$ observations, $X_{1}, \ldots, X_{n}$, typically one assumes that they are distributed independently according to some probability distribution with a finite mean and variance. For convenience, the Gaussian distribution is the most popular candidate; representing its mean and variance by $\mu$ and $\sigma^{2}$, it is well known that the ordinary sample mean and sample variance are "good" estimates, in that, on the average, they estimate $\mu$ and $\sigma^{2}$ unbiasedly and with minimum 
variance. Often, however, this Gaussian assumption is not exactly true, owing to a variety of reasons (e.g., measurement errors, outliers). Ideally, such departures from the assumed model should cause only small errors in the final conclusions. Such is not the case with the sample mean and sample variance; even one misspecified observation can throw these estimates far from the true $\mu$ and $\sigma^{2}$ (e.g., see Tukey's example in [7]).

It is important, then, to find alternative estimators of location and scale. Huber $[8$, p. 5] lists three desirable features of a statistical procedure:

1. reasonably efficient at the assumed model;

2. large changes in a small part of the data or small changes in a large part of the data should cause only small changes in the result (resistant);

3. gross deviations from the model should not severely decrease its efficiency (robust).

A class of estimators, called M-estimators, was proposed by Huber [9] to satisfy these three criteria. This class includes the sample mean in the following way. Let $T$ be the estimate which minimizes

$$
\sum_{i=1}^{n} \varrho\left(X_{\mathrm{i}}-T\right)
$$

where $\varrho$ is an arbitrary function. If $\Psi(x-\mu)=$ $(\partial / \partial \mu)_{Q}(x-\mu)$, then $T$ may also be defined implicitly by the equation

$$
\sum_{i=1}^{n} \Psi\left(X_{\mathrm{i}}-T\right)=0 \text {. }
$$

(There may be more solutions to $\left(1^{\prime}\right)$, however, corresponding to local minima of (1).)

If $\varrho(u)=\mathbf{u}^{2}$, then $(1)$ defines the sample mean $\bar{X}$ (and $\bar{X}$ is therefore called least squares estimate). It can be shown that M-estimates are maximum likelihood estimates (MLE) when $X_{1}, \ldots, X_{n}$ have a density proportional to $\exp \left\{-\int \Psi(\mathbf{u}) d u\right\}$ (e.g., $\vec{X}$ is MLE for the Gaussian distribution), but their real virtue is determined by their robustness in the face of possible departures from an assumed Gaussian model. Many suggestions for $\Psi$ have been offered, one of which is the biweight $\Psi_{-}$ function:

$$
\begin{aligned}
\Psi(\mathbf{u}) & =\mathbf{u}\left(1-\mathbf{u}^{2}\right)^{2} & & |\mathbf{u}| \leqslant 1 \\
& =0 & & \text { otherwise. }
\end{aligned}
$$

Using (2), $T$ as defined by ( $\left.1^{\prime}\right)$ is called the biweight. Actually, the solution in this form is not scale invariant.
We therefore define the biweight as the solution to the scale-invariant equation

$$
\sum_{\mathrm{i}=1}^{n} \Psi\left[\left(\mathrm{X}_{\mathrm{i}}-T\right) /(c \mathrm{~s})\right]=0,
$$

where $s$ is a measure of scale of the sample and $c$ is any positive constant, commonly called the "tuning constant." A graph of the biweight $\Psi$ function (2) is shown in figure 1 .

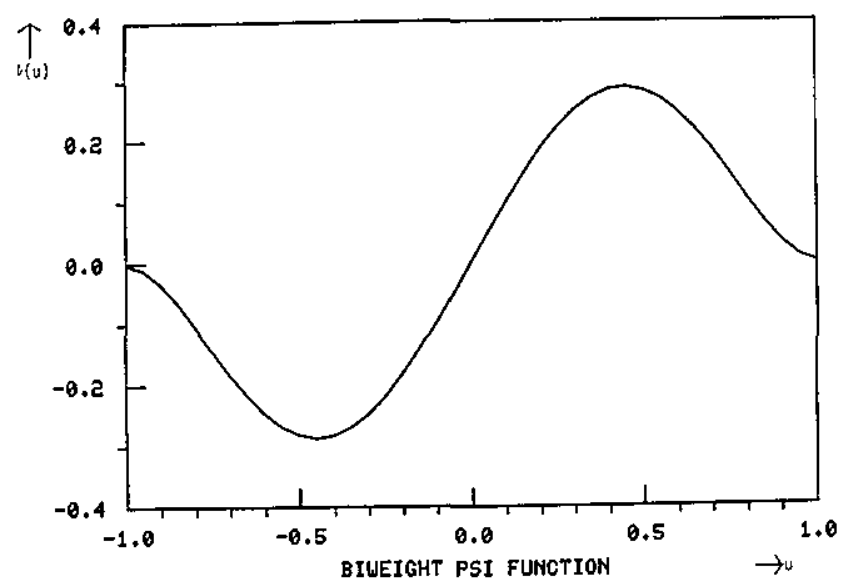

FIGURE 1

The lack of monotonicity in the biweight $\Psi$-function leads to its inclusion in the class of the so-called "redescending M-estimates," a term first introduced by Hampel [1, p. 14]. Typically, the defining $\Psi$-functions have finite support (i.e., are 0 outside a finite interval); hence, redescending $M$-estimates have the property that the calculation assigns zero weight to any observation which is more than $c$ multiples of the width from the estimated location. To see this, we define the weight function corresponding to any M-estimate, $w(\cdot)$, by the following equation:

$$
w(\mathrm{u})=\Psi(\mathrm{u}) / \mathrm{u} .
$$

Hence, (3) becomes

$$
0=\Sigma\left[\left(X_{\mathrm{i}}-T\right) /(c \mathrm{~s})\right] \cdot w\left[\left(X_{\mathrm{i}}-T\right) /(c \mathrm{~s})\right]
$$

which implies

$$
T=\Sigma X_{\mathbf{j}} w\left(\mathrm{u}_{\mathbf{i}}\right) / \Sigma w\left(\mathrm{u}_{\mathrm{i}}\right)
$$

where

$$
u_{i}=\left(X_{i}-T\right) /(c s)
$$


Equation (4) reveals that the calculation of $T$ may be viewed as an iteratively reweighted average of the observations. A graph of the weight function used for the biweight,

$$
\begin{aligned}
w(\mathbf{u}) & =\left(1-\mathbf{u}^{2}\right)^{2} & & |\mathbf{u}| \leqslant 1 \\
& =0 & & \text { otherwise, }
\end{aligned}
$$

also known as the bisquare weight function, is shown in figure 2 , where it is clear that zero weight is assigned to any value outside ( $T-c \mathrm{~s}, T+c \mathrm{~s})$. Henceforth, $\Psi$ and $w$ will always refer to the biweight $\mathrm{M}$-estimator.

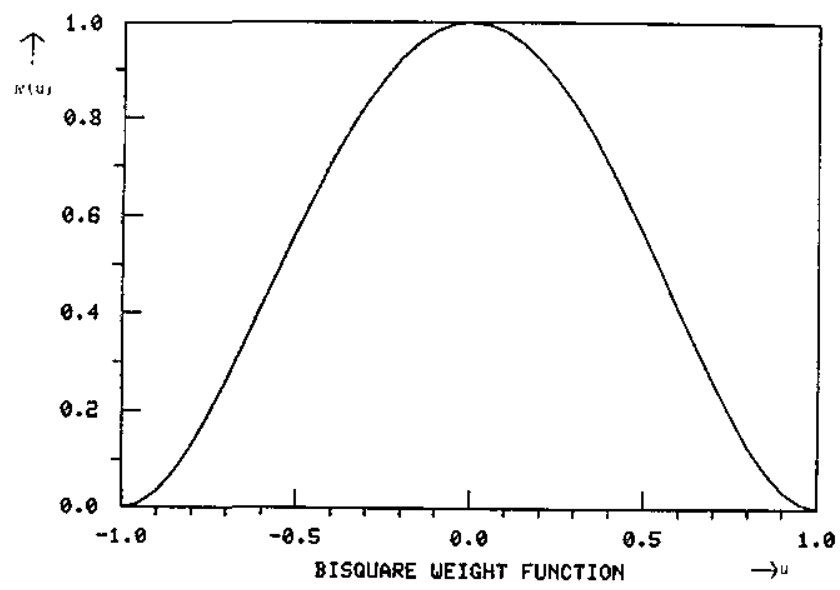

FIGURE 2

Because of the non-monotonicity of the biweight $\Psi$ function, multiple solutions to (3) are possible. It has been argued that an iteration based on (4) will not converge to all of the solutions to (3) and therefore will not get trapped by local minima of (1) [10]. In addition, the iteration suggested by eq (4) is more stable than a root finding search suggested by (3). These two facts encourage the use of (4), called the $w$-iteration, in calculating $T$.

\section{Use of the Biweight in Practice}

There has been considerable discussion on the practical usefulness of the biweight, and of redescending $M$ estimates in general. Huber points out that they are more sensitive to scaling (i.e., prior estimation of $s$ in (4)), and warns of possible problems in convergence $[8$, pp. 102-103]. In addition, unlike the monotone $\Psi_{-}$ functions, an estimate defined by a redescending $\psi_{-}$ function is not a maximum likelihood estimate for any density function, for it is constant outside a finite interval and hence does not integrate to 1 . The central (non- constant) part of what would be the corresponding density $\left(\exp \left(-\int \Psi(\mathrm{u}) \mathrm{du}\right)\right)$, scaled to have the same density at 0 as the unit Gaussian, reveals "shoulders" (Fig. 3), which may or may not correspond to realistic applications.

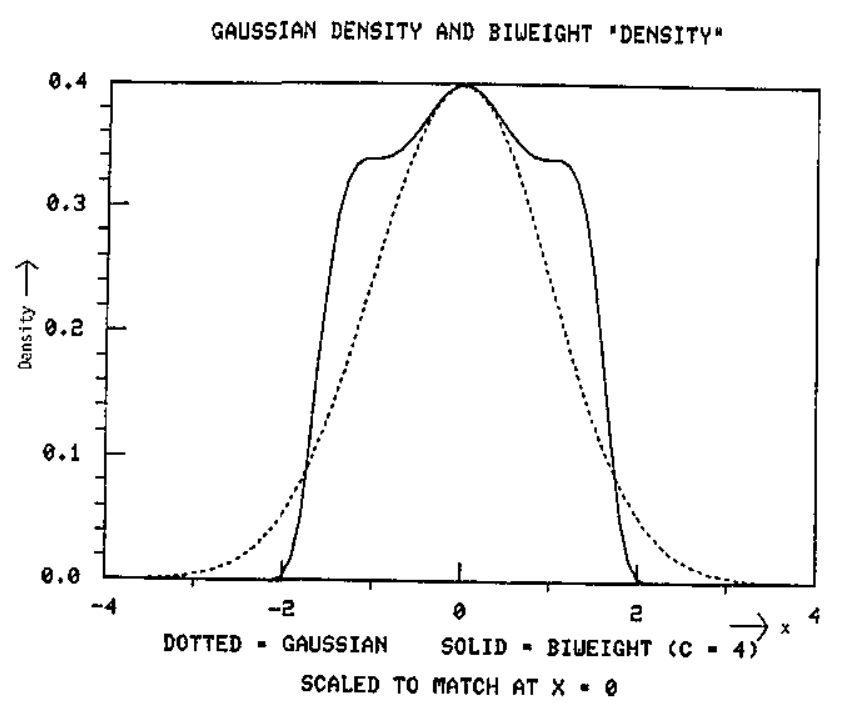

FIGURE 3

Nonetheless, the popularization of the biweight demands a careful assessment of its performance. This paper, therefore, documents its efficiency in three distributional situations using small- to moderate-sized samples.

The study reported below involved a Monte Carlo simulation of three situations, and three sample sizes, in order to determine the variance of the biweight using four different scalings and seven different values of the tuning constant. This section provides details on the calculation of the biweight, a description of the underlying situations in the Monte Carlo study, and the efficiency criterion on which it was evaluated.

\subsection{Calculation and Scalings}

Taking (3) as the definition of $T$ for this study, we calculate the biweight iteratively: after the $\mathrm{k}^{\text {th }}$ iteration,

$$
T^{(\mathrm{k}+1)}=\frac{\Sigma \mathrm{X}_{\mathrm{i}} w\left[\left(\mathrm{X}_{\mathrm{i}}-T^{(\mathrm{k})}\right) /(\mathrm{cs})\right]}{\Sigma w\left[\left(\mathrm{X}_{\mathrm{i}}-T^{(\mathrm{k})}\right) /(\mathrm{cs})\right]}, \quad \mathrm{k}=0,1,2 \ldots
$$

One may begin the iteration with any robust estimate of location. For this study, $T^{(0)}$ is the median for reasons of convenience and computational ease. In this form, the scale estimate remains fixed throughout the iteration. One may also consider updates on the scale: 


$$
T^{(\mathbf{k}+1)}=\frac{\sum \mathrm{X}_{\mathrm{i}} w\left[\left(\mathrm{X}_{\mathrm{i}}-T^{(\mathbf{k})}\right) /\left(\left(\mathrm{s}^{(\mathbf{k})}\right)\right]\right.}{\sum w\left[\left(\mathrm{X}_{\mathrm{i}}-T^{(\mathbf{k})}\right) /\left(c \mathrm{~s}^{(\mathbf{k})}\right)\right]}, \mathrm{k}=0,1,2 \ldots
$$

Two forms of scale functions were considered in connection with iterations (5) and (6). The median absolute deviation about the current estimate

$$
\mathrm{s}_{M A D}^{(k+1)}=\operatorname{med}_{1 \leqslant i \leqslant n}\left|\mathrm{X}_{\mathrm{i}}-T^{(k)}\right|, \quad T^{(0)}=\underset{1 \leqslant \mathrm{i} \leqslant \mathrm{n}}{\operatorname{med} \mathrm{X}_{\mathrm{i}}}
$$

or "MAD," has been used frequently in many robustness studies, including Andrews et al. [1]. In the Gaussian situation, the average value of the MAD is roughly two thirds of the standard deviation, so we really use $1.5 \times$ MAD. The second scale is based on a finite sample version of the theoretical asymptotic variance of $T[8$, p. 45]:

$$
\begin{aligned}
s_{b i}^{(k+1)} & =\left(\frac{n\left(c s_{b i}^{(k)}\right)^{2} \Sigma \Psi^{p}\left(u_{i}\right)}{\left[\Sigma \Psi^{\prime}\left(u_{i}\right)\right] \max \left[1,-1+\Sigma \Psi^{\prime}\left(u_{i}\right)\right]}\right)^{1 / 2} \\
u_{i} & =\left(X_{i}-T^{(k)}\right) /\left(c s_{b i}^{(k)}\right) .
\end{aligned}
$$

The subscript refers to the fact that $s_{b i}$ uses the bisquare weight function in its computation. The initial $s_{b i}{ }^{(0)}$, again for reasons of convenience, is taken here as $1.5 \times$ MAD. Equation (8) is designed to yield the ordinary sample variance when the $\Psi$-function is the identity (least squares); hence the use of the " -1 " in the denominator. Other values besides -1 have been investigated [1] but have proved less satisfactory. Equation (5) may also proceed without any scale updates (i.e., (7) and (8) calculated once and used throughout the iteration). Figure 4 illustrates four possibilities for scale evaluated in this study.

\subsection{Distributional Situations}

The variance of the biweight was calculated on three distributional situations:

- Gaussian ( $n$ observations from $\mathbf{N}(0,1)$ );

- One Wild ( $n-1$ observations from $N(0,1) ; 1$ unidentified observation from $\mathrm{N}(0,100))$;

- Slash ( $n$ observations from $N(0,1)$ /independent uniform on $[0,1])$.

The general term "situation" is applied particularly for the One-Wild, as the observations are not independent ( $n$-1 "reasonable-looking" observations suggest that the next is almost sure to be "wild"). The Slash distribution is a very stretched-tailed distribution like the Cauchy,

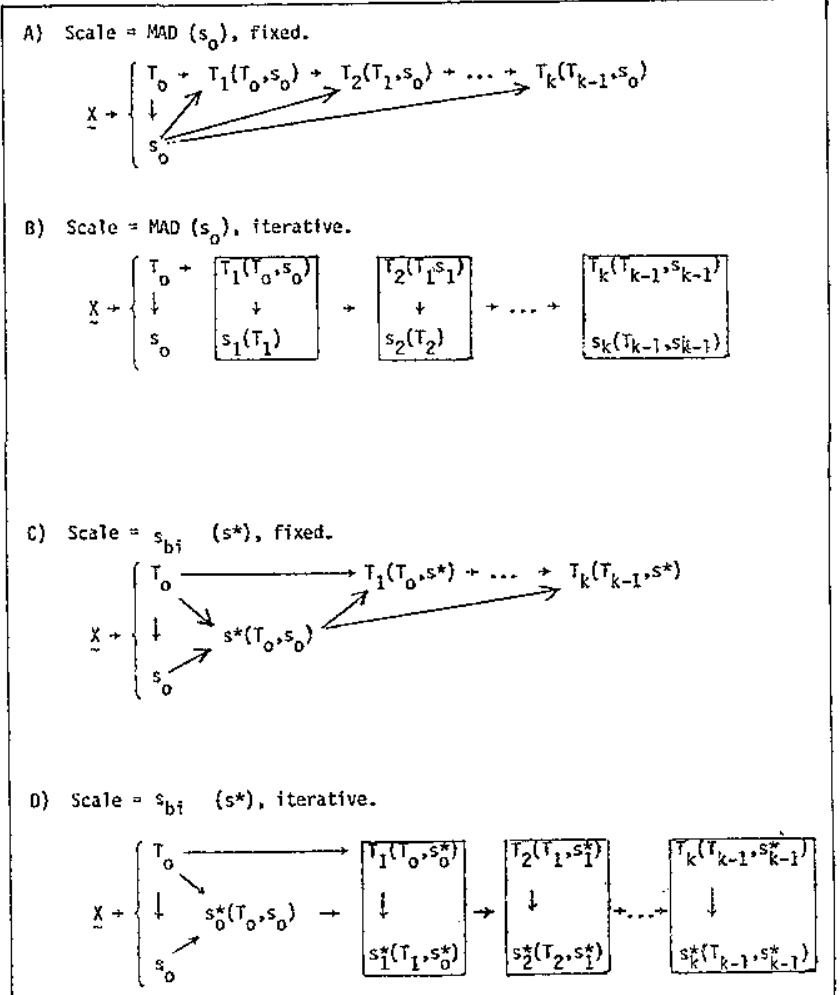

Figure 4. Four possible methods of iteration in the calculation of the biweight and associated scale from a sample $\underset{\sim}{\mathrm{X}}=\left(\mathrm{X}_{1}, \ldots\right.$, $\mathrm{X}_{\mathrm{n}}$ ) of $n$ observations.

For purposes of notational elarity, the following notation is used:

$$
\begin{aligned}
& \mathbf{T}=\text { biweight location estimate } \\
& \mathrm{s}=\text { MAD scale estimate (equation 7) } \\
& \mathbf{s}^{*}=\text { biweight scale estimate (equation } 8 \text { ) }
\end{aligned}
$$

and the subscript on each refers to the iteration at which the estimate is calculated.

but is less peaked in the center, making it a more realistic situation.

These three situations were chosen for two reasons. First, characteristics of sampling distributions of the various statistics may be estimated efficiently through a Monte Carlo swindle described by Simon [12] when the underlying distribution is of the form Gaussian/(symmetric positive distribution). Second, the three situations represent extreme types of situations for real-world applications ("utopian," outliers, and stretched tails); if an estimator performs well on these three, it is likely to perform well on almost any symmetric distribution arising in practice [13]. Additional characteristics about these distributions may be found in [14]. 


\subsection{Efficiency Comparisons}

In assessing the performance of a location estimator, one typically hopes for (i) unbiasedness, and (ii) minimal variance. It is simple to see that any M-estimate defined with an antisymmetric $\Psi$ function will be unbiased in symmetric situations. Furthermore, Huber has shown that under some regularity conditions, an M-estimator has an asymptotically Gaussian distribution with a finite variance, even for underlying distributions having infinite mean and variance [8, pp. 49-50]. Thus, it is reasonable to compare the variance of the biweight with the variance of the unbiased location estimator having minimal variance, if it exists, for a given situation.

It is known that the minimal variance that is attainable for an unbiased location estimator in the Gaussian situation is simply $1 / n$, or

$$
\operatorname{Var}(\sqrt{n} \bar{X})=V_{G}=1 .
$$

Minimal variances for the One-Wild and Slash, however, are not so simple. Theoretically, one might determine the variance of the maximum likelihood estimate for the One-Wild density but the derivation is not straightforward. A simple remedy is to pretend that one knows an observation is wild, which one it is, and eliminate it from the sample. Then the "near-optimal" variance would be

$$
\mathrm{V}_{\mathrm{w}}=n /(n-1)
$$

A "near-optimal" variance for the Slash density

$$
\begin{aligned}
(1 / \sigma) f(z)= & {\left[1-\exp \left(-z^{2} / 2\right)\right] /\left(\sqrt{2 \pi} \sigma z^{2}\right) } & & z \neq 0 \\
& (2 \sigma \sqrt{2 \pi})^{-1} & & z=0
\end{aligned}
$$

where

$$
z=(\mathrm{X}-\mu) / \sigma
$$

may be obtained through a maximum likelihood procedure. Details of this derivation may be examined in [15]. The variance of the Slash MLE, $V_{s}$, was determined within the Monte Carlo. For all three situations, the efficiency of the biweight is then calculated as

$$
\text { efficiency }=\frac{\text { "minimum" attainable variance }}{\text { variance (biweight) }} \text {. }
$$

Efficiency as close to 1 (or $100 \%$ ) as possible is desirable. So, sometimes it is more useful to calculate the complement, i.e., to examine how far

$$
\text { deficiency }=1 \text { - efficiency }
$$

is from zero (see $[1$, p. 121]).

\section{Results}

All computations were performed on a Univac 1108. One thousand samples of sizes 5,10 , and 20 were generated. Uniform deviates were obtained using a congruential generator [16]; the Box-Muller transform was applied to these to obtain Gaussian deviates [17]. The iteration in (4) was terminated when the relative change was less than 0.0005 , or if the number of iterations exceeded 15 (in which case, $T^{(15)}$ became the estimate of location).

Tables 1, 2, and 3 provide the variances, their sampling errors (SE) and deficiencies of the biweight for the Gaussian, One-Wild, and Slash situations. The most immediate observation is the low deficiency of the biweight in the Gaussian situation: using $c=4$, as recommended in Mosteller and Tukey [18], the biweight is never more than $10 \%$ less efficient than the optimal sample mean for any of the scalings here (except $n=5$, where it loses $15 \%$ for fixed $s_{b i}$ ). As $c$ increases, the deficiency is even lower. At $c=6$, even for $n=5$, the deficiency is less than $6 \%$. As noted by Mosteller and Tukey, relative differences in deficiency of less than $10 \%$ are essentially indistinguishable in practice $[18$, p. 206].

Comparing the scalings, $s_{b i}$ typically provides lower variances than does $1.5 \times$ MAD. The only exception to this is in some of the values computed for the Gaussian, where the differences are so small as to be unimportant (at $c=4$, largest difference $=4.9 \%$; at $c=6,2.7 \%$ ). The differences in deficiency can be quite sizeable for the One-Wild and Slash situations (e.g., at $c=4$, a difference of almost $20 \%$ for Slash, $n=20$ ).

In addition, one notes that the additional computation in updating the scale estimate with each iteration is not terribly worthwhile, as deficiencies are only trivially higher in most cases. In fact, such updating can cause considerable deficiency. As a check on the convergence of the iteration, table 4 shows the number of samples, out of 1000 , that did not satisfy the convergence criterion. Most of the non-convergences occurred with the iterative scales, particularly the iterative MAD. 
TABLE 1. Biweight variances and deficiencies: $n=5$.

\begin{tabular}{|c|c|c|c|c|c|c|c|c|c|c|c|c|}
\hline \multirow[b]{2}{*}{$\begin{array}{l}\text { Tuning } \\
\text { Constant }\end{array}$} & \multicolumn{3}{|c|}{ 1.5xMAD - Fixed } & \multicolumn{3}{|c|}{ 1.5xMAD - Iterative } & \multicolumn{3}{|c|}{$s_{b i}-$ Fixed } & \multicolumn{3}{|c|}{$s_{b i}-$ Iterative } \\
\hline & Variance & SE & $\begin{array}{c}\text { Deficiency } \\
(\%)\end{array}$ & Variance & SE & $\begin{array}{c}\text { Deficiency } \\
(\%)\end{array}$ & Variance & SE & $\begin{array}{c}\text { Deficiency } \\
(\%)\end{array}$ & Variance & $\mathrm{SE}$ & $\begin{array}{c}\text { Deficiency } \\
(\%)\end{array}$ \\
\hline \multicolumn{13}{|c|}{ Gaussian (optimal $=1.0$ ) } \\
\hline 3 & 1.2114 & 0.0172 & 17.5 & 1.1959 & 0.0171 & 16.4 & 1.2652 & 0.0203 & 21.0 & 1.2475 & 0.0201 & 21.1 \\
\hline 4 & 1299 & 0134 & 11.5 & 1065 & 0130 & 9.6 & 1694 & 0163 & 14.5 & 1294 & 0149 & 12.6 \\
\hline 5 & 0879 & 0109 & 8.1 & 0702 & 0106 & 6.6 & 1068 & 0132 & 9.6 & 0816 & 0126 & 8.6 \\
\hline 6 & 0611 & 0088 & 5.8 & 0398 & 0082 & 3.8 & 0693 & 0106 & 6.5 & 0550 & 0104 & 6.1 \\
\hline 7 & 0438 & 0072 & 4.2 & 0200 & 0053 & 2.0 & 0487 & 0090 & 4.6 & 0371 & 0086 & 4.4 \\
\hline 8 & 0326 & 0061 & 3.2 & 0155 & 0045 & 1.5 & 0387 & 0084 & 3.7 & 0311 & 0082 & 3.8 \\
\hline 9 & 0264 & 0057 & 2.6 & 0124 & 0043 & 1.2 & 0262 & 0070 & 2.6 & 0204 & 0068 & 2.6 \\
\hline \multicolumn{13}{|c|}{ One-Wild (optimal $=1.2$ ) } \\
\hline 3 & 1.7377 & 0.0314 & 30.9 & 1.7064 & 0.0302 & 29.7 & 1.6946 & 0.0282 & 29.2 & 1.6979 & 0.0281 & 30.5 \\
\hline 4 & 2.0164 & 0461 & 40.4 & 2.1890 & 0658 & 45.2 & 1.8322 & 0366 & 34.5 & 1.9621 & 0447 & 40.2 \\
\hline 5 & 2.4523 & 0709 & 51.1 & 3.0922 & 1281 & 61.2 & 2.1110 & 0540 & 43.2 & 2.5201 & 0835 & 53.9 \\
\hline 6 & 2.9642 & 0977 & 59.5 & 4.2740 & 1933 & 71.9 & 2.6146 & 0850 & 54.1 & 3.3286 & 1302 & 65.3 \\
\hline 7 & 3.5080 & 1235 & 65.8 & 5.6037 & 2657 & 78.6 & 3.2118 & 1199 & 62.6 & 4.2358 & 1748 & 72.8 \\
\hline 8 & 4.0822 & 1481 & 70.6 & 6.9447 & 3101 & 82.7 & 3.9072 & 1537 & 69.3 & 5.0837 & 2163 & 77.4 \\
\hline 9 & 4.6817 & 1725 & 74.4 & 8.3881 & 3705 & 85.7 & 4.5843 & 1877 & 73.8 & 5.9725 & 2561 & 80.7 \\
\hline \multicolumn{13}{|c|}{ Slash loptimal $=10.375\}$} \\
\hline 3 & 22.291 & 5.787 & 53.4 & 21.964 & 6.008 & 52.8 & 22.497 & 6.346 & 53.9 & 22.312 & 5.976 & 63.3 \\
\hline 4 & 23.470 & 6.034 & 55.8 & 33.974 & 11.664 & 69.5 & 22.618 & 6.006 & 54.1 & 30.965 & 10.533 & 75.0 \\
\hline 5 & 25.218 & 6.331 & 58.8 & 37.209 & 12.131 & 72.1 & 26.191 & 7.061 & 60.4 & 33.872 & 11.295 & 77.0 \\
\hline 6 & 28.207 & 6.981 & 63.2 & 42.045 & 12.466 & 75.3 & 31.643 & 9.610 & 67.2 & 37.952 & 11.764 & 79.1 \\
\hline 7 & 31.873 & 3.205 & 67.4 & 45.744 & 12.647 & 77.3 & 34.461 & 10.723 & 69.9 & 39.713 & 12.034 & 80.0 \\
\hline 8 & 34.778 & 9.289 & 70.2 & 47.387 & 12.743 & 78.1 & 37.223 & 11.570 & 72.1 & 42.677 & 12.312 & 81.1 \\
\hline 9 & 36.791 & 10.073 & 71.8 & 52.963 & 13.121 & 80.4 & 39.923 & 12.188 & 74.0 & 45.549 & 12.470 & 82.1 \\
\hline
\end{tabular}

Figures 5, 6, and 7 provide graphs of deficiency as a function of the tuning constant, for sample sizes $n=5$, 10 , and 20 . The uncertainty limits on these graphs, plotted with dotted lines, are given by

$$
1 \text { - ["minimum" variance]/[var(biweight }) \pm \mathrm{SE}] \text {, }
$$

where $\mathrm{SE}$ refers to the Monte Carlo sampling error in the calculation of the biweight variances. These reveal that, across these three situations, $c=4$ to $c=6$ is a practical value of the tuning constant, for larger values tend to yield extremely high deficiencies for the Slash.

The biweight deficiencies computed here scaled by 1.5 $x$ MAD (fixed) differ from those computed by Holland and Welsch [19] in part because of the difference in starting value (they took $T^{(0)}=$ least absolute deviations estimate), and in convergence criterion (they took $T^{(5)}$ as their solution). Asymptotically, $c=4.685$ yields $95 \%$ asymptotic efficiency at the Gaussian [i.e., $\sqrt{n} T$ con- verges in distribution to $\mathrm{N}(0,1.0526)]$; within two sampling errors, the results in tables 1,2 , and 3 are consistent with this value.

As noted earlier, $T^{(15)}$ became the estimate of location in cases of non-convergence in the study. This only increases the variance of the biweight that we are likely to see in practice, because this situation occurred typically when the iteration alternated between two equally distant values from $\mu$. In practice, one should examine the sample to determine the cause of non-convergence, and possibly settle on the median and $1.5 \times$ MAD as expedient location and scale estimates.

\section{An Example}

To illustrate the calculation of the biweight, we use some chemical measurements collected at the National Bureau of Standards. These data were taken from 
TABLE 2. Biweight variances and deficiencies: $n=10$.

\begin{tabular}{|c|c|c|c|c|c|c|c|c|c|c|c|c|}
\hline \multirow{3}{*}{$\begin{array}{l}\text { Tuning } \\
\text { Constant }\end{array}$} & \multicolumn{3}{|c|}{ 1.5xMAD - Fixed } & \multicolumn{3}{|c|}{ 1.5xMAD - Iterative } & \multicolumn{3}{|c|}{$s_{b i}-$ Fixed } & \multicolumn{3}{|c|}{$s_{b i}-$ Iterative } \\
\hline & & & $\overline{\text { Deficiency }}$ & & & ficiency & & & Deficiency & & & Deficiency \\
\hline & Variance & SE & $(\%)$ & Variance & SE & $(\%)$ & Variance & SE & $(\%)$ & Variance & SE & $(\%)$ \\
\hline \multicolumn{13}{|c|}{ Gaussian (optimal $=1.0$ ) } \\
\hline 3 & 1.1250 & 0.0109 & 11.1 & 1.1083 & 0.0115 & 9.8 & 1.1937 & 0.0146 & 16.2 & 1.1930 & 0.0156 & 16.2 \\
\hline 4 & .0578 & 0075 & 5.5 & 0469 & 0069 & 4.5 & 0961 & 0099 & 8.8 & 0800 & 0083 & 7.4 \\
\hline 5 & 0319 & 0056 & 3.1 & 0236 & 0051 & 2.3 & 0479 & 0068 & 4.6 & 0335 & 0048 & 3.2 \\
\hline 6 & 0198 & 0043 & 1.9 & 0133 & 0035 & 1.3 & 0259 & 0048 & 2.5 & 0145 & 0032 & 1.4 \\
\hline 7 & 0126 & 0031 & 1.2 & 0074 & 0026 & 0.7 & 0145 & 0034 & 1.4 & 0056 & 0013 & 0.6 \\
\hline 8 & 0084 & 0024 & 0.8 & 0039 & 0017 & 0.4 & 0078 & 0023 & 0.8 & 0037 & 0013 & 0.4 \\
\hline 9 & 0059 & 0019 & 0.6 & 0029 & 0019 & 0.3 & 0042 & 0015 & 0.4 & 0027 & 0013 & 0.3 \\
\hline \multicolumn{13}{|c|}{ One-Wild (optimal $=1.1111$ ) } \\
\hline 3 & 1.2785 & 0.0116 & 13.1 & 1.2781 & 0.0114 & 13.1 & 1.3414 & 0.0161 & 17.2 & 1.3413 & 0.0168 & 17.2 \\
\hline 4 & 1.2946 & 0109 & 14.1 & 1.3105 & 0117 & 15.2 & 2709 & 0112 & 12.6 & 1.2630 & 0105 & 12.0 \\
\hline 5 & 1.3714 & 0137 & 19.0 & 1.4125 & 0159 & 21.3 & 2775 & 0104 & 13.0 & 1.2828 & 0102 & 13.4 \\
\hline 6 & 1.4990 & 0189 & 25.9 & 1.5948 & 0246 & 30.3 & 3257 & 0115 & 16.2 & 1.3794 & 0141 & 19.4 \\
\hline 7 & 1.6793 & 0253 & 33.8 & 1.8333 & 0332 & 39.4 & 4196 & 0163 & 21.7 & 1.5757 & 0242 & 29.5 \\
\hline 8 & 1.9034 & 0331 & 41.6 & 2.1332 & 0445 & 47.9 & 5621 & 0223 & 28.9 & 1.9037 & 0397 & 41.6 \\
\hline 9 & 2.1590 & 0420 & 48.5 & 2.4703 & 0580 & 55.0 & 7562 & 0301 & 36.1 & 2.3703 & 0591 & 53.1 \\
\hline \multicolumn{13}{|c|}{ Slash (optimal $=5.9843$ ) } \\
\hline 3 & 7.0895 & 0.2795 & 15.6 & 7.4991 & 0.3348 & 20.2 & 6.4815 & 0.2422 & 7.7 & 6.5367 & 0.2465 & 8.5 \\
\hline 4 & 7.9896 & 3382 & 25.1 & 8.6854 & 0.4129 & 31.1 & 7.1538 & .2796 & 16.3 & 7.6037 & 0.3339 & 21.3 \\
\hline 5 & 8.9977 & 4355 & 33.5 & 10.819 & 0.9005 & 44.7 & 8.0679 & 3586 & 25.8 & 9.2930 & 0.7666 & 35.6 \\
\hline 6 & 10.261 & 5815 & 41.7 & 14.567 & 1.4310 & 58.9 & 8.9836 & 4739 & 33.4 & 11.167 & 0.8863 & 46.4 \\
\hline 7 & 11.487 & 7135 & 47.9 & 25.156 & 8.6783 & 76.2 & 10.555 & 7319 & 43.3 & 22.739 & 7.8504 & 73.7 \\
\hline 8 & 12.692 & 8214 & 52.8 & 28.036 & 9.0950 & 78.6 & 11.705 & 5120 & 51.6 & 28.258 & 8.5066 & 78.8 \\
\hline 9 & 13.999 & 9294 & 57.3 & 33.752 & 9.4 .908 & 82.3 & 12.908 & 6051 & 58.4 & 31.727 & 8.9310 & 81.1 \\
\hline
\end{tabular}

several ampoules of $n-H e p t a n e$ material at NBS between May 22 and June 17, 1981. The ampoules were filled from two lots in several sets. Lot $A$ includes 20 sets of ampoules; lot $B$ includes six sets; only the data from 10 ampoules in sets from lot $A$ will be used here. Panel $A$ of table 5 shows the mean percent purity from 10 ampoules, where the mean was calculated as an average of anywhere between 5 and 10 measurements. To eliminate the big numbers and decimal points, we subtract 99.9900 and multiply by $10^{4}$ in the third column.

Notice that, by virtue of the central limit theorem, one would expect that these averages would be approximately normally distributed and a higher value for the tuning constant, say $c=5$, would be reasonable. The third column of Panel A reveals three somewhat anomolous values: $-20,56$, and 28 . Notice that the low value corresponds to ampoules in set 2, and the high value to those in set 20 . Since the sets were filled sequentially, there may have been some aspect of the filling procedure which caused these odd values. Also, the data are listed in the order in which they were measured, so the low value for the first ampoule may have resulted from some problem in the measuring equipment on the first day.

The iteration initiates with the median, $T^{(0)}=7$, and $s_{b i}$ is calculated from the median and $1.5 \mathrm{x}$ MAD $(=12.0)$, yielding a scale estimate of 17.2 . The convergence criterion in this calculation is relative to the estimated scale; i.e., the iteration ceases when either $k \geqslant$ 15 or $\left|T^{(\mathbf{k})}-T^{(\mathbf{k}-1)}\right| / s_{b i} \leqslant .0005$.

Panel B gives the bisquare weights associated with each observation and the biweight at each iteration. Notice that the three "suspect" values all receive lower weight than the other seven. The final scale estimate, 0.0019 , is computed from the final location estimate, 99.9907, and the $s_{b i}$ used throughout the iteration (.0017). These estimates compare favorably with the sample mean and standard deviation, 99.9910 and 0.0021 . 
TABLE 3. Biweight variances and deficiencies: $n=20$.

\begin{tabular}{|c|c|c|c|c|c|c|c|c|c|c|c|c|}
\hline \multirow{2}{*}{$\begin{array}{l}\text { Tuning } \\
\text { Constant }\end{array}$} & \multicolumn{3}{|c|}{ 1.5xMAD - Fixed } & \multicolumn{3}{|c|}{ 1.5xMAD - Iterative } & \multicolumn{3}{|c|}{$s_{b i}-$ Fixed } & \multicolumn{3}{|c|}{$s_{b i}-$ Iterative } \\
\hline & Variance & SE & $\begin{array}{c}\text { Deficiency } \\
(\%)\end{array}$ & Variance & SE & $\begin{array}{c}\text { Deficiency } \\
(\%)\end{array}$ & Variance & SE & $\begin{array}{c}\text { Deficiency } \\
(\%)\end{array}$ & Variance & SE & $\begin{array}{c}\text { Deficiency } \\
(\%)\end{array}$ \\
\hline \multicolumn{13}{|c|}{ Gaussian (optimal $=1.0$ ) } \\
\hline 3 & 1.0973 & 0.0076 & 8.8 & 1.0841 & 0.0069 & 7.8 & 1.2111 & 0.0147 & 17.4 & 1.2159 & 0.0154 & 17.8 \\
\hline 4 & 0369 & 0039 & 3.6 & 0299 & 0034 & 2.9 & .0842 & 0064 & 7.8 & 0769 & 0051 & 7.1 \\
\hline 5 & 0172 & 0026 & 1.7 & 0117 & 0013 & 1.2 & 0387 & 0036 & 3.7 & 0331 & 0024 & 3.2 \\
\hline 6 & 0087 & 0015 & 0.9 & 0056 & 0007 & 0.6 & 0187 & 0019 & 1.8 & 0163 & 0015 & 1.6 \\
\hline 7 & 0047 & 0008 & 0.5 & 0030 & 0004 & 0.3 & 0096 & 0010 & 0.9 & 0084 & 0008 & 0.8 \\
\hline 8 & 0027 & 0005 & 0.3 & 0018 & 0002 & 0.2 & 0052 & 0005 & 0.5 & 0045 & 0004 & 0.4 \\
\hline 9 & 0017 & 0003 & 0.2 & 0011 & 0001 & 0.1 & 0030 & 0003 & 0.3 & 0027 & 0002 & 0.3 \\
\hline \multicolumn{13}{|c|}{ One-Wild (optimal $=1.0526$ ) } \\
\hline 3 & 1.1597 & 0.0077 & 9.2 & 1.1494 & 0.0055 & 8.4 & 1.2663 & 0.0148 & 16.9 & 1.2561 & 0.0139 & 16.2 \\
\hline 4 & 1313 & 0040 & 7.0 & 1298 & 0037 & 6.8 & 1517 & 0066 & 8.6 & 1421 & 0053 & 7.8 \\
\hline 5 & 1572 & 0049 & 9.0 & 1594 & 0050 & 9.2 & 1198 & 0034 & 6.0 & 1185 & 0034 & 5.9 \\
\hline 6 & 2082 & 0069 & 12.9 & 2145 & 0071 & 13.3 & 1273 & 0037 & 6.6 & 1289 & 0037 & 6.8 \\
\hline 7 & 2745 & 0094 & 17.4 & 2848 & 0097 & 18.1 & 1522 & 0047 & 8.6 & 1578 & 0050 & 9.1 \\
\hline 8 & 3532 & 0122 & 22.2 & 3721 & 0127 & 23.2 & 1905 & 0062 & 11.6 & 2019 & 0067 & 12.4 \\
\hline 9 & 4439 & 0151 & 27.1 & 4690 & 0159 & 28.3 & 2431 & 0081 & 15.3 & 2700 & 0092 & 17.1 \\
\hline \multicolumn{13}{|c|}{ Slash (optimal $=5.2666$ ) } \\
\hline 3 & 6.2724 & 0.2046 & 16.0 & 6.4046 & 0.2284 & 17.8 & 5.6057 & 0.1410 & 6.1 & 6.7146 & 0.1541 & 7.8 \\
\hline 4 & 7.5085 & 3189 & 29.9 & 8.1706 & 0.4386 & 35.5 & 6.2212 & 1976 & 15.3 & 6.4293 & 0.2137 & 18.1 \\
\hline 5 & 8.8490 & 4364 & 40.5 & 10.255 & 0.6849 & 48.6 & 7.3065 & 2822 & 27.9 & 8.3053 & 0.4552 & 36.6 \\
\hline 6 & 10.157 & 5377 & 48.1 & 12.260 & 0.8502 & 57.0 & 8.6312 & 4237 & 39.0 & 10.434 & 0.6585 & 49.5 \\
\hline 7 & 11.453 & 6307 & 54.0 & 13.964 & 1.0004 & 62.3 & 10.116 & 5670 & 47.9 & 12.580 & 0.8205 & 58.1 \\
\hline 8 & 12.733 & 7235 & 58.6 & 15.762 & 1.1107 & 66.6 & 11.832 & 7166 & 55.4 & 15.324 & 1.0068 & 65.7 \\
\hline 9 & 13.996 & 8083 & 62.3 & 17.100 & 1.1866 & 69.2 & 13.442 & 8405 & 60.8 & 18.235 & 1.1790 & 71.1 \\
\hline
\end{tabular}

In this case, there is little difference between the two procedures, and either may be reported. Had there been a substantial difference, one would want to examine the data more closely to understand the reason. This is an important step in data analysis, and robust methods offer easy, objective procedures for making this comparison and illustrating possible anomolies in the data.

\section{Conclusions}

This paper establishes the variance of the biweight as a location estimator across three distributional situations, for small to moderate sample sizes. In terms of scaling, $s_{b i}$ performs more satisfactorily than does $1.5 \times \mathrm{MAD}$, and need not be recalculated with subsequent iterations.
Three to six iterations of the $w$-iteration are typically required to attain satisfactory convergence $\left(<.0005 \times s_{b i}\right)$. The minimum efficiencies of the biweight across the three situations for sample sizes, 5,10 , and 20 at $c=4$ are $46 \%$, $83 \%$, and $85 \%$ respectively; at $c=6$ they are $33 \%, 67 \%$, and $61 \%$ respectively. Gaussian efficiencies are considerably higher: at $c=4,86 \%, 91 \%$, and $92 \%$; at $c$ $=6,94 \%, 97 \%$, and $98 \%$.

A final comment concerns the results on $n=5$. For such a small sample size, it is encouraging that the biweight $(c=4$ ) is only $14 \%$ less efficient than the optimal sample mean if the underlying population is really Gaussian. In fact, $s_{b i}$ can be very misleading in a small (between $2 \%$ and $5 \%$ for the situations listed here) but influential proportion of the time. Conditioning on some ancillary statistic, such as the average value of the weights, would undoubtedly increase the efficiency for all three situations when $n=5$. 
TABLE 4. Number of samples (out of 1000 samples of size $n=5 / n=10 / n=20)$ that did not converge $\left(k>15\right.$ and $\left|T^{(k+1)}\right|>$ $.0005 \times$ scale).

\begin{tabular}{|c|c|c|c|c|}
\hline $\begin{array}{c}\text { (Tuning } \\
\text { Constant) }\end{array}$ & $\begin{array}{l}\text { 1.5xMAD } \\
\text { FIXED }\end{array}$ & $\begin{array}{c}\text { 1.5xMAD } \\
\text { ITERATIVE }\end{array}$ & $\underset{\text { FIXED }}{s_{b i}}$ & $\stackrel{s_{b i}}{\text { ITERATIVE }}$ \\
\hline \multicolumn{5}{|l|}{ Gaussian } \\
\hline $\mathbf{3}$ & $6 / 0 / 0$ & $106 / 44 / 9$ & $1 / 0 / 0$ & $34 / 13 / 0$ \\
\hline 4 & $9 / 0 / 0$ & $92 / 5 / 1$ & $1 / 0 / 0$ & $0 / 6 / 1$ \\
\hline 5 & $1 / 0 / 0$ & $68 / 8 / 2$ & $1 / 0 / 0$ & $0 / 2 / 0$ \\
\hline 6 & $5 / 0 / 0$ & $50 / 4 / 0$ & $0 / 0 / 0$ & $0 / 2 / 0$ \\
\hline 7 & $3 / 0 / 0$ & $33 / 2 / 0$ & $1 / 0 / 0$ & $0 / 0 / 0$ \\
\hline 8 & $2 / 0 / 0$ & $29 / 2 / 0$ & $0 / 0 / 0$ & $0 / 0 / 0$ \\
\hline 9 & $0 / 0 / 0$ & $20 / 2 / 0$ & $0 / 0 / 0$ & $0 / 0 / 0$ \\
\hline \multicolumn{5}{|l|}{ One-Wild } \\
\hline $\mathbf{3}$ & $3 / 0 / 0$ & $120 / 11 / 6$ & $0 / 0 / 0$ & $18 / 13 / 0$ \\
\hline 4 & $1 / 0 / 0$ & $168 / 14 / 3$ & $0 / 0 / 0$ & $0 / 10 / 3$ \\
\hline 5 & $1 / 0 / 0$ & $170 / 42 / 0$ & $0 / 0 / 0$ & $0 / 9 / 0$ \\
\hline 6 & $0 / 0 / 0$ & $159 / 70 / 3$ & $0 / 0 / 0$ & $0 / 47 / 0$ \\
\hline 7 & $1 / 0 / 0$ & $138 / 91 / 6$ & $0 / 0 / 0$ & $0 / 46 / 0$ \\
\hline 8 & $0 / 0 / 0$ & $121 / 113 / 20$ & $0 / 0 / 0$ & $0 / 36 / 0$ \\
\hline 9 & $0 / 0 / 0$ & $92 / 120 / 37$ & $0 / 0 / 0$ & $0 / 22 / 32$ \\
\hline \multicolumn{5}{|l|}{ Slash } \\
\hline 3 & $4 / 0 / 0$ & $119 / 31 / 20$ & $1 / 0 / 0$ & $0 / 0 / 15$ \\
\hline 4 & $5 / 0 / 0$ & $156 / 33 / 16$ & $0 / 0 / 0$ & $1 / 3 / 15$ \\
\hline 5 & $1 / 0 / 0$ & $138 / 51 / 24$ & $0 / 0 / 0$ & $0 / 0 / 41$ \\
\hline 6 & $8 / 0 / 0$ & $121 / 81 / 36$ & $1 / 0 / 0$ & $0 / 0 / 94$ \\
\hline 7 & $1 / 0 / 0$ & $99 / 65 / 39$ & $1 / 0 / 0$ & $0 / 0 / 56$ \\
\hline 8 & $0 / 0 / 0$ & $79 / 64 / 48$ & $0 / 0 / 0$ & $0 / 0 / 76$ \\
\hline 9 & $0 / 0 / 0$ & $67 / 53 / 53$ & $0 / 0 / 0$ & $0 / 32 / 74$ \\
\hline
\end{tabular}

DEFICIENCIES OF BIWEIGHT, $N \cdot 5$

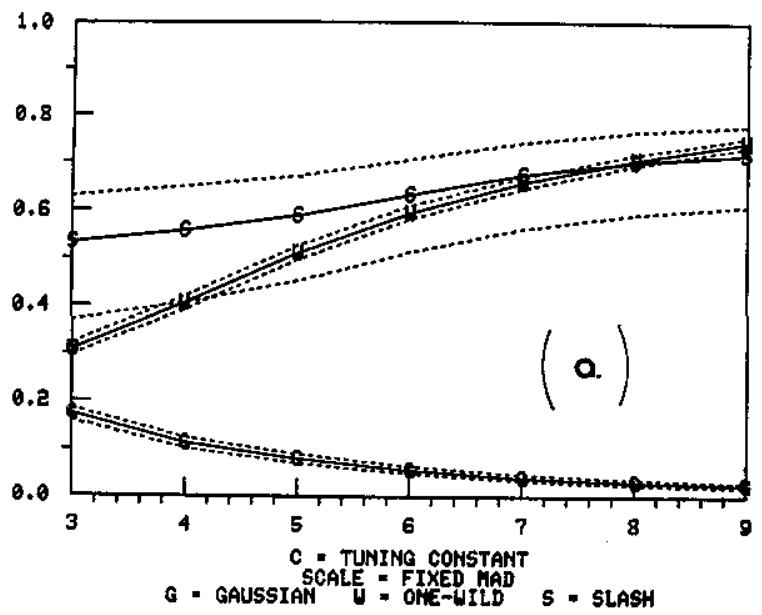

Figure 5(a)
DEFICIFNCIES OF BIUEIGHT, $N$ = 5

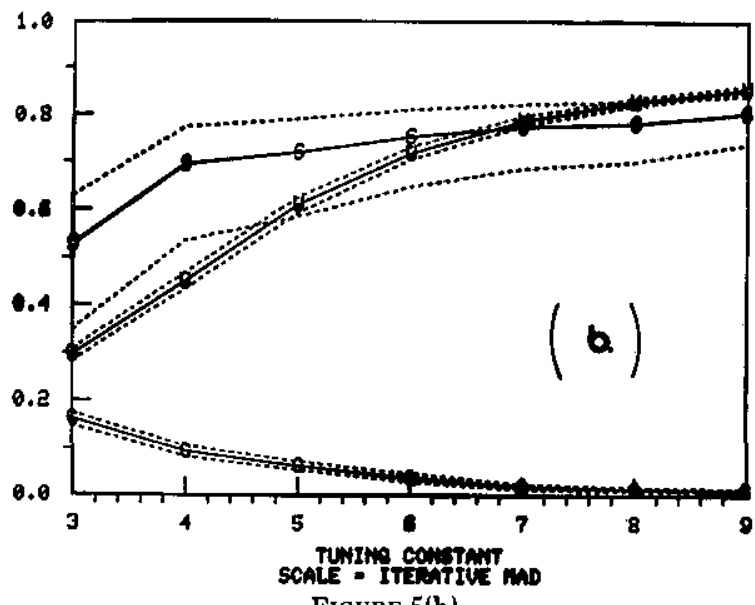

Figure 5(b)

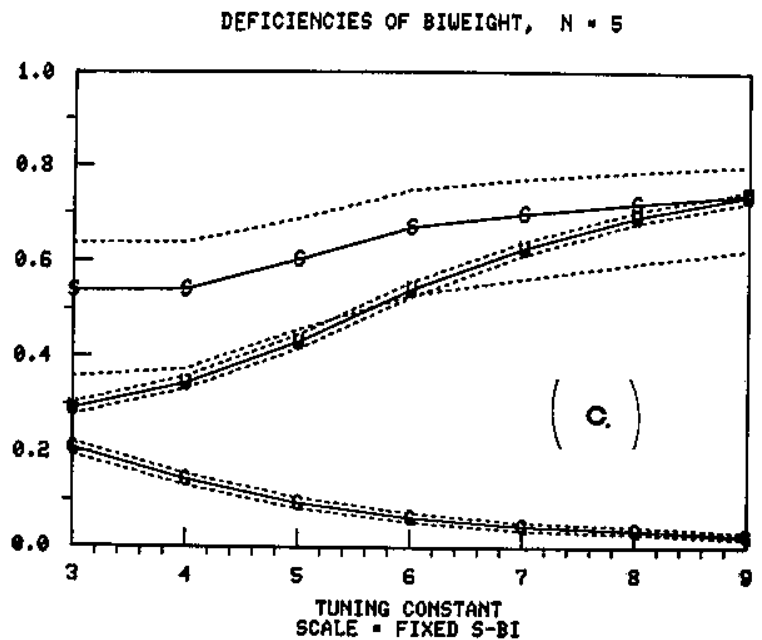

Figure 5(c)

DEFICIENCIES OF BIWEIGHT, $N=5$

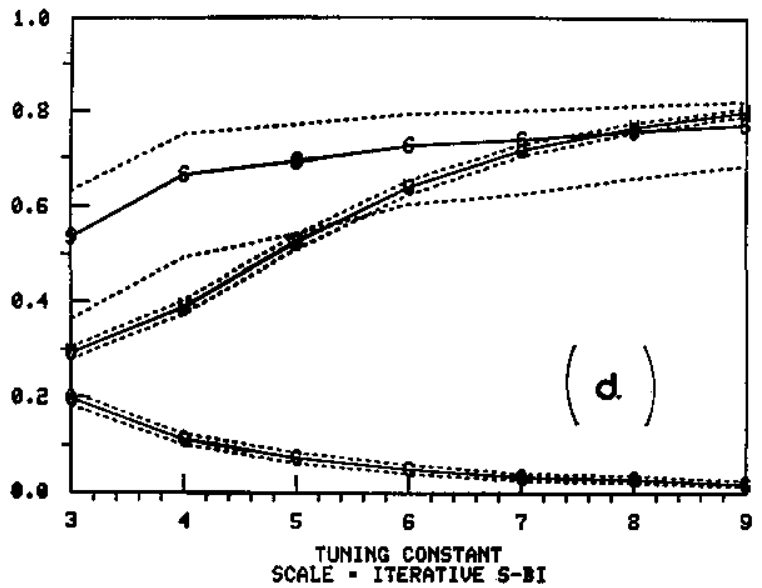

Figure 5(d) 
DEFICIENCIES OF BIWEIGHT, $N=10$

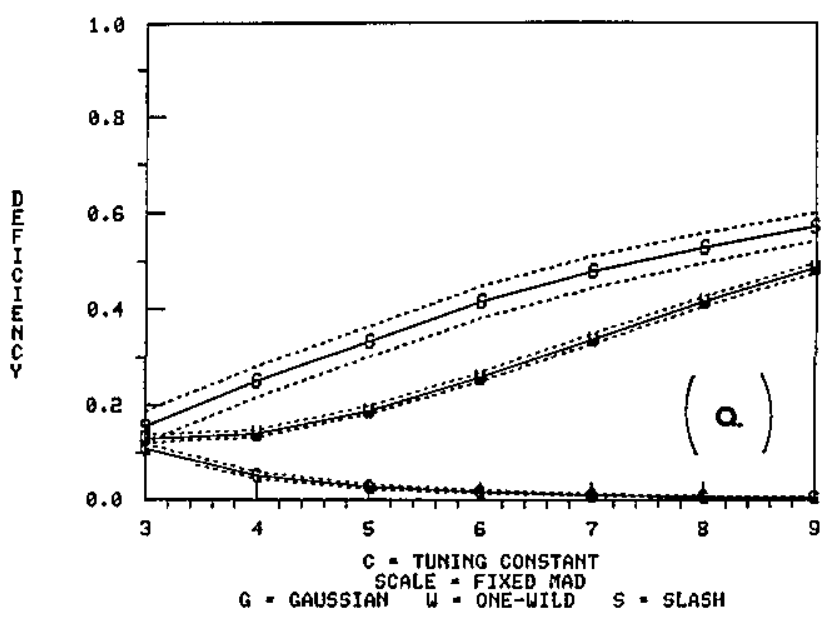

Figure 6(a)

DEFICIENCIES OF BIWEIGHT, $N=10$

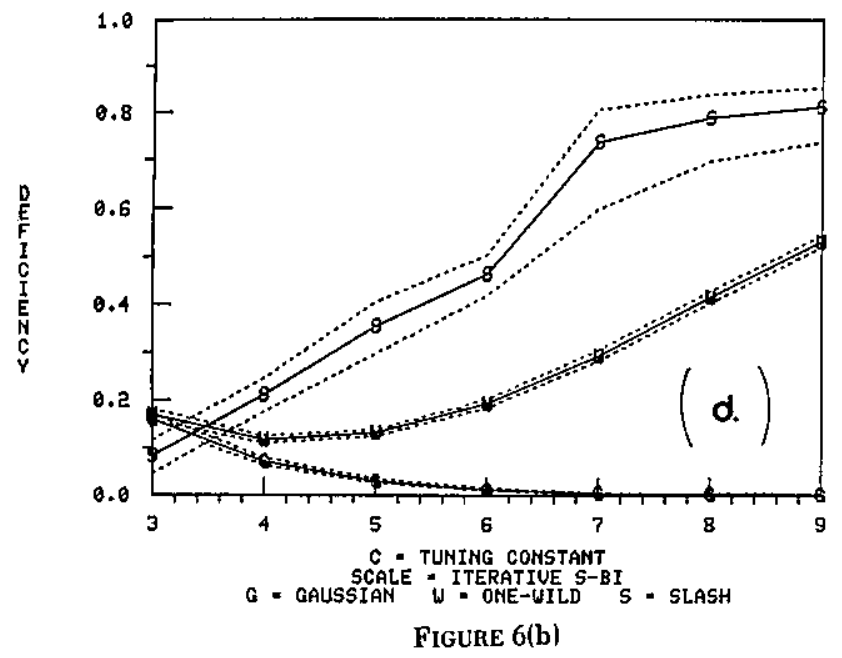

DEFICIENCIES OF BIWEIGHT, $N=10$

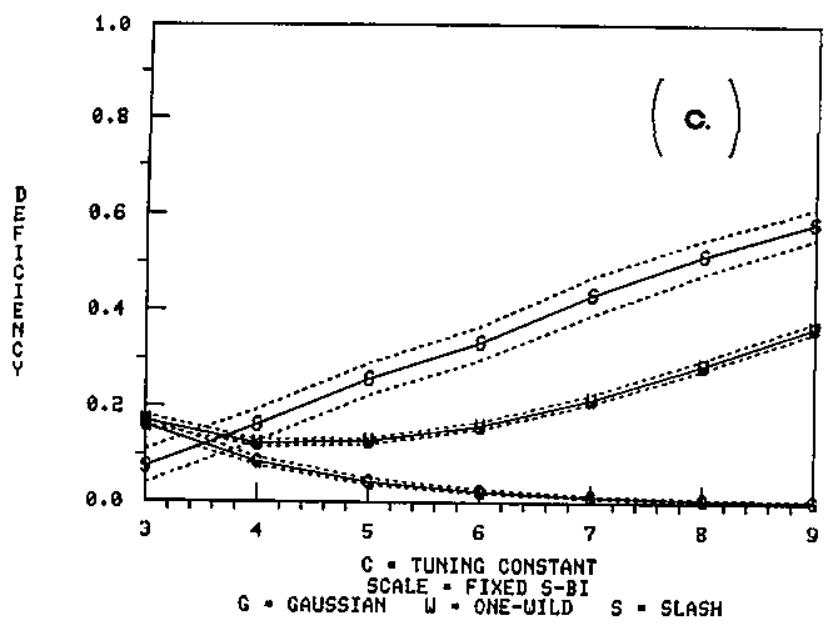

Figure 6(c)
DEFICIENCIES OF BIUEIGHT, $N=10$

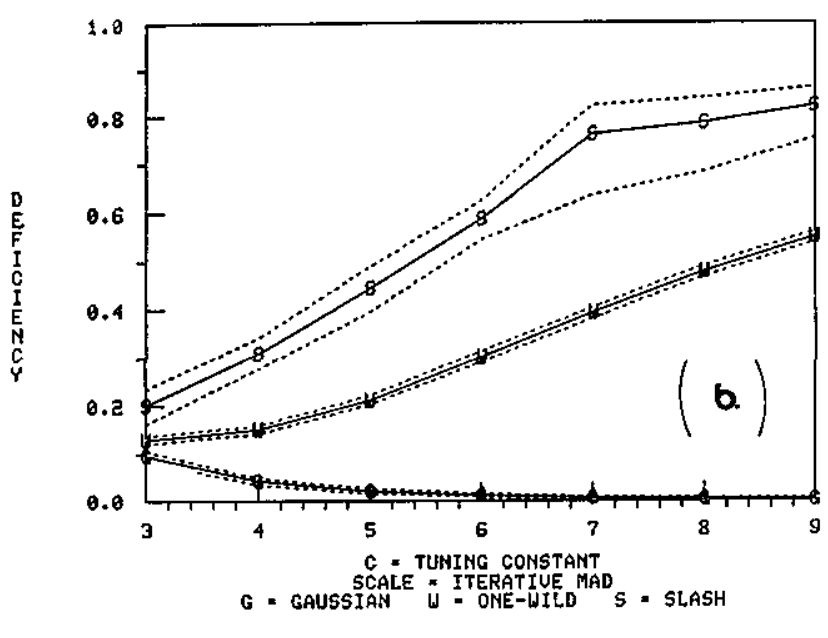

Figure 6(d)

DEFICIENCIES OF BIWEIGHT, $N=20$

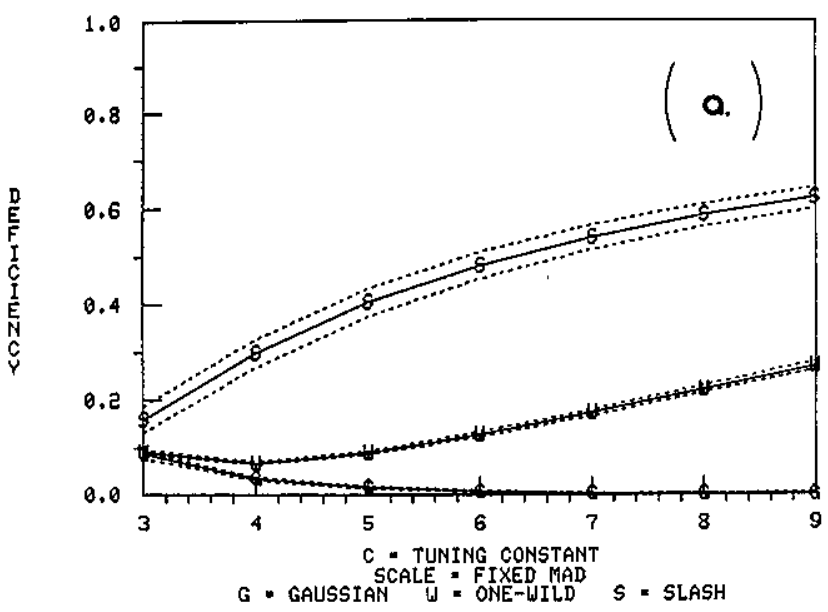

FIGURE 7(a)

DEFICIENCIES OF BIUEIGHT, $N=20$

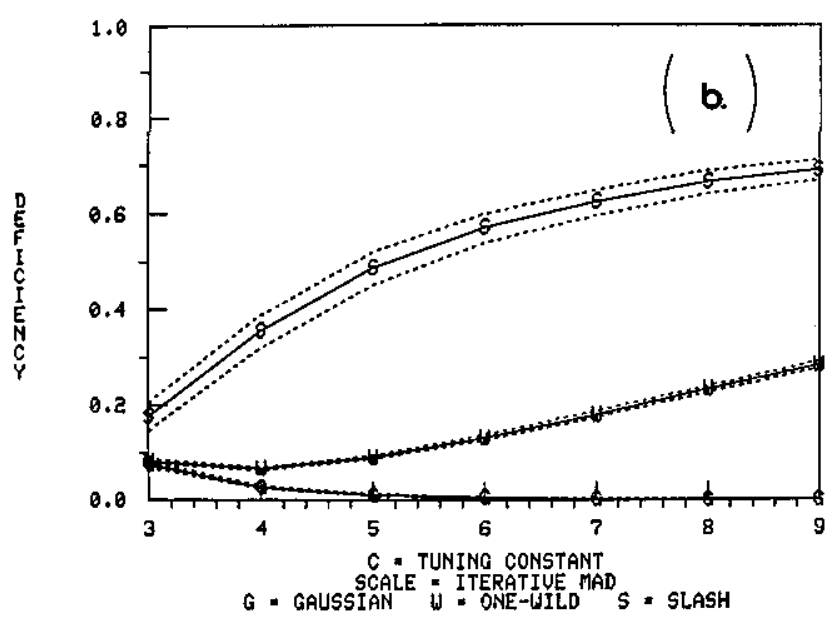

Figure 7(b) 
DEFICIENCIES OF BIWEIGHT, $N \cdot 20$

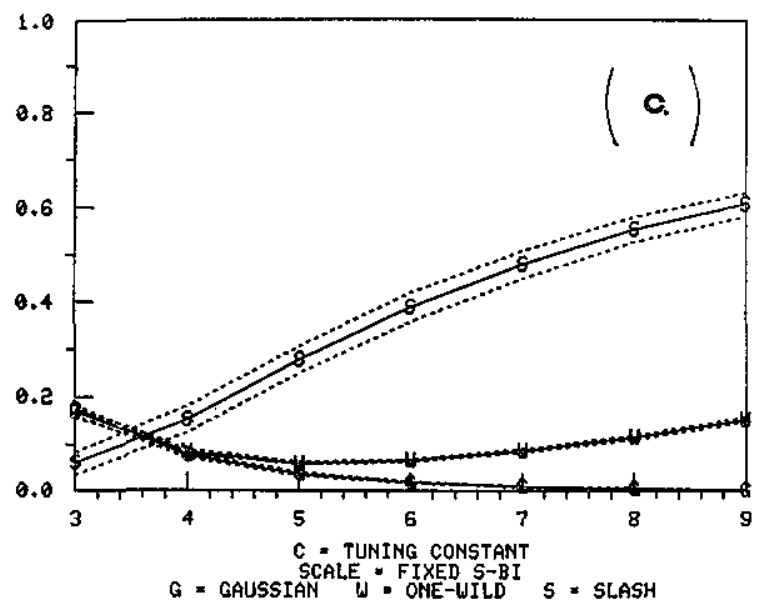

Figure 7(c)

DEFICIENCIES OF BIWEIGHT, $N \cdot 20$

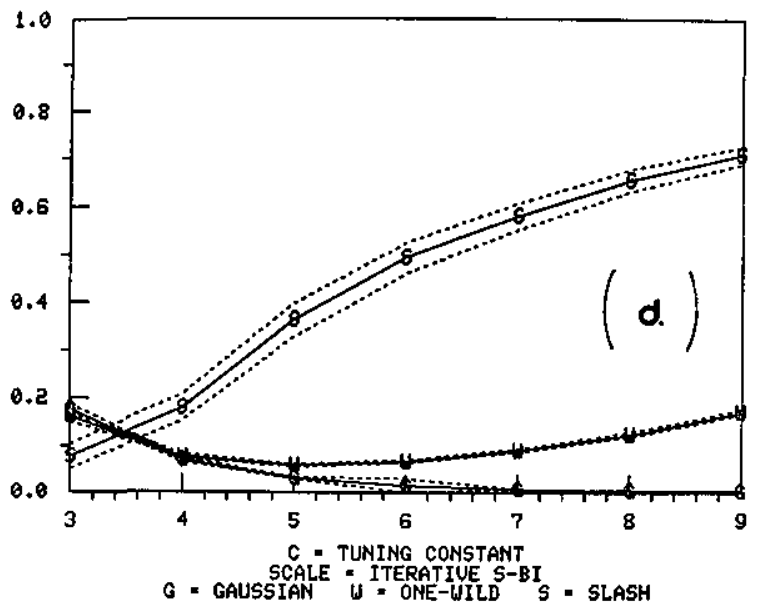

Figure 7 (d)

\section{References}

[1] Andrews, D.F.; Bickel, P.J.; Hampel, F.R.; Huber, P.J.; Rogers, W.H.; and Tukey, J.W. (1972). Robust Estimates of Location: Survey and Advances, Princeton University Press: Princeton, New Jersey.

[2] Gross, A.M., and Tukey, J. W. The estimators of the Princeton Robustness Study. Technical Report No. 38, Series 2, Department of Statistics, Princeton University, Princeton, New Jersey.

[3] Beaton, A.E., and Tukey, J.W. (1974). The fitting of power series, meaning polynomials, illustrated on band-spectroscopic data. Technometrics 16, 147-185.

[4] Gross, Alan M. (1976). Confidence interval robustness with longtailed symmetric distributions. J. Amer. Statist. Assoc. 71, 409-417.
TABLe 5. Measurements on ampoules of n-Heptane.

\begin{tabular}{|c|c|c|}
\hline A) The da & & \\
\hline $\begin{array}{l}\text { Set No.- } \\
\text { Ampoule }\end{array}$ & $\%$ purity & (\% purity -99.99$) \cdot 10^{4}$ \\
\hline $2-05$ & 99.9880 & -20 \\
\hline $3-04$ & 99.9909 & 9 \\
\hline $20-20$ & 99.9956 & 56 \\
\hline $7-07$ & 99.9908 & 8 \\
\hline $20-03$ & 99.9901 & 1 \\
\hline $14-10$ & 99.9928 & 28 \\
\hline $2-15$ & 99.9915 & 15 \\
\hline $8-14$ & 99.9899 & -1 \\
\hline 14-20 & 99.9906 & 6 \\
\hline $7-17$ & 99.9894 & -6 \\
\hline
\end{tabular}

B) Biweight iterations: $c=5, T^{(0)}=7.0, s^{(0)}=12.0, s_{b i}=17.2$

Columns give weights $w_{i}^{(k)}$ corresponding to each observation $y_{i}$ at $\mathrm{k}^{\text {th }}$ iteration. $T^{(k)}=\Sigma w_{i}^{(k)} y_{i} / \Sigma w_{i}^{(k)}$

\begin{tabular}{|c|c|c|c|c|c|}
\hline obsv'n & (1) & (2) & (3) & (4) & (5) \\
\hline-20 & .8120 & .8082 & .8075 & .8074 & .8074 \\
\hline 9 & .9989 & .9992 & .9992 & .9993 & .9993 \\
\hline 56 & .4546 & .4597 & .4606 & .4607 & .4608 \\
\hline 8 & .9997 & .9999 & .9999 & 9999 & .9999 \\
\hline 1 & .9903 & .9893 & .9891 & .9891 & .9891 \\
\hline 28 & .8839 & .8869 & .8875 & .8876 & .8876 \\
\hline 15 & .9827 & .9839 & .9841 & .9842 & .9842 \\
\hline-1 & .9827 & .9815 & .9812 & .9812 & .9812 \\
\hline 6 & 9997 & .9996 & .9995 & .9995 & .9995 \\
\hline-6 & .9547 & .9527 & .9523 & .9523 & .9523 \\
\hline
\end{tabular}

Final location and scale estimates (original scale):

$$
\begin{array}{lrl}
\hat{\mu} & =99.9900+7.346 / 10^{4}= & 99.9907 \\
\hat{o}= & 18.648 / 10^{4}= & .0019
\end{array}
$$

[5] Gross, Alan M. (1977). Confidence intervals for bisquare regression estimates. J. Amer. Stat. Assoc. 72, 341-354.

[6] Kafadar, K. (1982b). Using biweights in the two-sample problem. Comm. Statist. 11(17), 1883-1901.

[7] Tukey, J.W. (1960). A survey of sampling from contaminated distributions. In Contributions to Probability and Statistics, I. Olkin, ed., Stanford University Press, Stanford, California.

[8] Huber, P. (1981). Robust Statistics. Wiley: New York.

[9] Huber, P. (1964). Robust estimation of a location parameter. Ann. Math. Statist. 35, 73-101.

[10] Lax, D. (1975). An interim report of a Monte Carlo study of robust estimates of width. Technical Report No. 93, Series 2, Department of Statistics, Princeton University, Princeton, New Jersey. 
[11] Tukey, J.W.; Braun, H.I., and Schwarzchild, M. (1977). Further progress on robust/resistant widths. Technical Report No. 129, Series 2, Department of Statistics, Princeton University, Princeton, New Jersey.

[12] Simon, G. (1976). Computer simulation swindles with applications to estimates of location and dispersion. Appl. Statist. 25, 266-274.

[13] Tukey, J.W. (1977). Lecture Notes to Statistics 411. Unpublished manuscript, Princeton University, Princeton, New Jersey.

[14] Rogers, W.F., and Tukey, J.W. (1972). Understanding some longtailed symmetrical distributions. Statistica Neerlandica 26, No. $3,211-226$.
[15] Kafadar, K. (1982a). A biweight approach to the one-sample problem. J. Amer, Stat. Assoc. 77, 416-424.

[16] Kronmal, J. (1964). Evaluation of the pseudorandom number generator. J. Assoc. Computing Machinery, 351-363.

[17] Box, G.E.P., and Muller, M. (1958). A note on generation of normal deviates. Ann. Math. Stat. 28, 610-611.

[18] Mosteller, F., and Tukey, J.W. (1977). Data Analysis and Regression: $A$ Second Course in Statistics. Addison-Wesley; Reading, Massachusetts.

[19] Holland, P.W., and Welsch, R.E. (1977). Robust regression using iteratively reweighted least squares. Comm. Statist. A6(9), 813-827. 